Ulises Sepúlveda, Felipe Portales, Francisco Maturana: Experiencias de resistencia y agencia espacio-laboral de trabajadores de un barrio tradicional frente a cambios globalizadores: el Barrio Victoria, Santiago de Chile, Izquierdas, 49, julio 2020:1177-1197

\title{
Experiencias de resistencia y agencia espacio-laboral de trabajadores de un barrio tradicional frente a cambios globalizadores: el Barrio Victoria, Santiago de Chile
}

\section{Experiences of resistance and spatial-labor agency of workers from traditional neighborhoods facing} globalizing changes. The case of Barrio Victoria, Santiago de Chile

Ulises Sepúlveda S.*

Felipe Portales O.*

Francisco Maturana M.***

Resumen: La globalización y la apertura económica de un país generan nuevos desafíos para los espacios comerciales locales y tradicionales. El presente trabajo aborda esta problemática situándola en un espacio comercial típico de Santiago: el barrio Victoria; vinculado al rubro del cuero y el calzado. Se busca comprender las experiencias de resistencia y la agencia espacio-laboral de los trabajadores frente al fenómeno de importación de calzado asiático. Para responder a ello se dispuso de un método cualitativo/espacial que promueve un análisis relacional de los contextos laborales presentes en el barrio, a partir de la agencia espacio-laboral que efectúan los locatarios. Se exponen dos tipos de resultados. El primero corresponde a las prácticas de resiliencia y/o reelaboración implementadas por los sujetos estudiados, y el segundo dilucida las "micro geografías de producción y reproducción laboral" y sus distintas configuraciones espaciales. Se sugiere que los locatarios se han logrado adecuar a las nuevas dinámicas de producción y demanda por medio de nuevas lógicas de trabajo, las cuales desarrollaron una segmentación en el rubro del cuero y gestaron una articulación de espacios de fabricación y venta final.

Palabras claves: agencia, globalización, barrio Victoria, espacios laborales, análisis cualitativo

\begin{abstract}
Globalization and the economic opening of a country generate new challenges for local and traditional commercial spaces. The present work addresses this problem placing it in a typical commercial space of Santiago: Victoria neighborhood; linked to the field of leather and footwear. The aim is to understand the subsistence and adaptation of this commercial space in the face of the phenomenon of the importation of Asian footwear. To respond to this, a qualitative/spatial method was used to promote a relational analysis of the work spaces present in the neighborhood, based on the space-work agency carried out by tenants.
\end{abstract}

Keywords: agency, globalization, barrio Victoria, work spaces, qualitative analysis.

Recibido: 16 enero 2019 Aceptado: 3 abril 2019

* Chileno, Coautor, Departamento de Geografía, Universidad Alberto Hurtado, usepulve@uahurtado.cl

** Chileno, Coautor, Departamento de Geografía, Universidad Alberto Hurtado, fportale@alumnos.uahurtado.cl

${ }^{* * *}$ Chileno, Coautor, Departamento de Geografía, Universidad Alberto Hurtado, fmaturana@uahurtado.cl 
Ulises Sepúlveda, Felipe Portales, Francisco Maturana: Experiencias de resistencia y agencia espacio-laboral de trabajadores de un barrio tradicional frente a cambios globalizadores: el Barrio Victoria, Santiago de Chile, Izquierdas, 49, julio 2020:1177-1197

\section{Introducción}

Las reestructuraciones económicas globales y sus impactos en distintos espacios locales alrededor del mundo se han comenzado a estudiar en las últimas décadas desde las geografías económicas con enfoque social (Carswell y De Neve, 2013; Coe y Hess, 2013). Así, definir la relación global-local, se hace necesaria para comprender los lugares; espacios locales, que comienzan a tener una mayor interacción con escalas geográficas más amplias, dentro de este proceso de reestructuración económica y social. Massey realiza un aporte significativo al proponer un sentido global del lugar que viene a comprender la especificidad de estos, como "momentos articulados en redes de relaciones, experiencias, e interpretaciones sociales en los que una gran proporción de estas relaciones, experiencias e interpretaciones están construidas a una escala mucho mayor que la que define en aquel momento el sitio mismo, sea una calle, una región o incluso un continente" (Massey, 1991, 28).

Esta conceptualización, destaca la naturaleza procesual de los lugares, como entes activos y nunca cerrados. Así, sería posible mirar el espacio de barrio en la ciudad en un contexto socio-espacial, donde a través de sus relaciones con otros espacios, genera una particularidad. Siguiendo a Massey (1991), correspondería a un punto de encuentro en donde distintas relaciones sociales se han entretejido. La misma autora abarca, desde una perspectiva relacional, los espacios de producción y el trabajo, por medio de las Divisiones espaciales del trabajo (Massey, 1995). Así, los principales aspectos de la reestructuración económica se vincularían a las relaciones de producción a distintas escalas, que vienen a incidir directamente en un espacio. Este (considerado acá como un barrio) laboral-comercial, se ve incidido por estos componentes estructurales que trasforman los espacios locales desde la industrialización y que se acentúan en el proceso globalizador.

Las relaciones sociales (de producción) dentro de este marco de globalización son desiguales, existen relaciones de poder en donde diferentes grupos e individuos están situados de maneras muy diferentes ante estos flujos e interconexiones (Massey, 1993), lo que la misma autora ha denominado geometrías del poder. Estas relaciones desiguales, también abarcan a los espacios laborales y comerciales, generando diferentes posiciones de poder. Un ejemplo clarificador de aquello, son las diferencias que se pueden observar entre los grandes centros comerciales y los espacios comerciales locales que ofrecen productos similares y, por ende, atienden a una misma tipología de consumidores.

Los grandes centros comerciales (malls), son proyectos de inversiones transnacionales o grandes firmas nacionales que son expresiones de lo que se ha denominado como compresiones de espacio-tiempo, las cuales son "provocadas por producciones de espacio y cambios tecnológicos que hacen posible que la comunicación sea lanzada y abaratada, de modo que los horizontes de tiempo de la toma de decisiones se reducen, mientras que los efectos de estas decisiones rebotan en un espacio cada vez más amplio y diferenciado" (Harvey, 1998).

Los efectos materiales de esta compresión espacio-tiempo, conjugan en un mismo contexto una gran oferta y variedad de productos, afectando directamente a espacios comerciales especializados, que responden a otro ritmo de producción y relación escalar. Estas geometrías de poder también revelan en muchos casos una relación dialéctica: compresión espacio-tiempo/ expansión espacio-tiempo propuesta por Katz (2004), en donde la expansión surge como una respuesta de la primera. En efecto, la expansión espacio-temporal apreciable sería una consecuencia local de esta comprensión espacio-tiempo, es decir, lo que en palabras de Pumain y Saint-Julien (2014) es una contracción espacio temporal que permite el desarrollo de mayores interacciones producto de las mejoras en tecnología, disminución de costos de transporte y donde 
Ulises Sepúlveda, Felipe Portales, Francisco Maturana: Experiencias de resistencia y agencia espacio-laboral de trabajadores de un barrio tradicional frente a cambios globalizadores: el Barrio Victoria, Santiago de Chile, Izquierdas, 49, julio 2020:1177-1197

finalmente existe un mayor movimiento de flujos, tanto en velocidad como intensidad, lo cual se ha visto acrecentado por el proceso de globalización.

Tomando en cuenta el contexto señalado, el presente trabajo tiene por objetivo comprender las formas de agencia espacio laboral de los trabajadores del barrio Victoria, en Santiago de Chile. Se enfoca la investigación desde una perspectiva cualitativa, utilizando muestreo intencional (Patton, 1995) y entrevistas topológicas (Sepúlveda, 2018). Como producto, se señalan dos tipos de resultados, los de primer orden vinculados a cambios en las lógicas de trabajo mediadas por prácticas de resiliencia y/o reelaboración y, los de segundo orden, vinculados a las micro geografías de producción y reproducción laboral. Estas últimas, dan cuenta de las estrategias desarrolladas por estos actores en relación con el embate neoliberal, los cuales resultan ejemplos ilustrativos de como los comercios locales han logrado subsistir al impacto sistémico.

\section{La geografía laboral y la perspectiva de la agencia como una forma de abordar las repercusiones de la globalización en espacios locales}

En geografía humana contemporánea, se han tomado en cuenta diferentes perspectivas y componentes estructurales a la hora de estudiar las repercusiones de la globalización en espacios locales. Una de estas perspectivas se centra en el estudio de las geografías minoristas, las cuales surgen debido a "una creciente comprensión entre los científicos sociales de la concentración progresiva de capital en la industria minorista, el aumento muy visible de la corporación minorista (la 'megacadena' como Walmart en los Estados Unidos y Tesco en el Reino Unido), la creciente importancia que tales corporaciones habían comenzado a asumir en varias economías occidentales, y el surgimiento de corporaciones minoristas como firmas líderes" (Wrigley, 2009). Desde estas geografías minoristas, emerge un nuevo campo que se vincula al estudio de la sociedad de consumo y la cultura del consumidor.

Las investigaciones relacionadas a las geografías de consumo, buscan conjugar los aspectos económicos de la globalización y los impactos que estos tienen en las prácticas culturales (Goodman, Goodman, y Redclift, 2010; Jackson, 2004; Mansvelt, 2005; Miller et al. 1998). Por otro lado, encontramos las geografías laborales que, en la actualidad, ponen atención a las experiencias y acciones, tanto de trabajadores individuales, como de colectividades que se adecúan a las nuevas espacialidades laborales que responden a dinámicas globales.

En tal contexto, las relaciones globales-locales vivenciadas por los trabajadores y sus formas de "ganarse la vida", han sido conceptualizadas en varias ocasiones mediante la agencia laboral para ofrecer una visión del trabajador o locatario como un sujeto autónomo. Este tendría la capacidad de generar estrategias, que producen efectos transformadores en sus medios de subsistencia, relaciones sociales y capacidad reproductiva de los espacios en los cuales desarrollan su trabajo (Carswell y De Neve, 2013). En ese sentido, esta geografía busca desarrollar una comprensión más fundamentada de la agencia y las acciones de los trabajadores (Dutta, 2016).

\section{Apertura económica en Chile: importaciones asiáticas y su incidencia en el barrio}

Los procesos acá mencionados, se han desarrollado en varios lugares del mundo. En este sentido, Chile no ha quedado ajeno y su impacto más importante es posible percibirlo en Santiago, la cual sufrió una verdadera re estructuración económica a partir de las políticas implementadas tras el golpe de Estado (De Mattos, 1999) y que fue profundizada en los gobiernos democráticos posteriores (Fuentes y Sierralta, 2004). En efecto, las reestructuraciones económicas realizadas en el período en la década de 1970, 
Ulises Sepúlveda, Felipe Portales, Francisco Maturana: Experiencias de resistencia y agencia espacio-laboral de trabajadores de un barrio tradicional frente a cambios globalizadores: el Barrio Victoria, Santiago de Chile, Izquierdas, 49, julio 2020:1177-1197

desencadenan un impacto certero en diferentes barrios de la ciudad y en distintas áreas industriales ${ }^{1}$, destacando la industria del cuero nacional con el establecimiento del modelo económico neoliberal, que comienza a configurar nuevas relaciones de producción y pautas de consumo en el país.

Lo anterior, ha sido muy bien relatado por Kassai $(2000,12)$, el cual indica que "el shock importador que se produjo como consecuencia de la disminución de los aranceles a partir de 1974, originó un aumento considerable del déficit comercial y una reducción de la producción interna con efectos negativos en los mercados respectivos. A modo de ejemplo, el desequilibrio en la balanza comercial del calzado aumentó de -0.8 a -57.1 millones de dólares, mientras el del cuero y marroquinería pasó de -1.0 a -30.1 millones de dólares entre 1967 y $1981 "$.

Con el ingreso del modelo económico neoliberal, las condiciones de la fuerza de trabajo también se ven afectadas "en una etapa de internacionalización de los procesos productivos en base a la búsqueda de mano de obra barata" (Lastra, 2018, 258). Esto se tradujo en una baja sistemática de los salarios percibidos por los trabajadores industriales del país. Asimismo, la pérdida de estabilidad laboral durante la época, permitió la conformación de nuevos actores sociales (como las asalariadas agrícolas en Chile) que luchan por mejorar las condiciones precarias en las que desarrollan su trabajo (Valdés, Godoy \& Mendoza, 2017).

Es en tal contexto, que uno de los barrios que vio reconfigurado su espacio y relaciones productivas corresponde al barrio Victoria (ver su localización en figura 1). Tal sector, se constituyó hace más de cien años como un espacio comercial en un contexto muy diferente al actual. Estaba "tradicionalmente vinculado a la industria del cuero, en cuanto a su confección, producto de la localización del tradicional matadero Franklin hacia el año 1847 en donde se generaba la materia prima necesaria para la curtiembre" (Sandoval, 2012, 153) y se caracterizaba por ser un lugar de residencia-trabajo, es decir, los trabajadores residían en las cercanías de sus puestos de trabajo, en su mayoría.

Entre los años 1895 al 1918, el peso relativo del sector cuero representaba un 9,3\% de la producción de todos los sectores industriales y artesanales de Chile. Sólo era superado por el sector alimenticio (45\%), el de la madera $(12 \%)$ y el textil $(10 \%)$ (Carmagnani, 1998). Dada la convergencia de trabajadores que desarrollan sus labores en un mismo espacio, se comenzó a reconocer una especialización de las actividades comerciales en este barrio y otros cercanos (San Diego, 10 de julio, Franklin, entre otros) en una época de auge de un modelo de sustitución de importaciones que fomentaba las actividades industriales y manufactureras (Palma, 1984).

Así, el barrio Victoria, se establecía como uno de los espacios comerciales más importantes en Santiago, en donde se congregaban una gran cantidad de las denominadas suelerías, que vienen a atender las necesidades de artesanos del cuero en busca de materiales (distintos tipos de cuero) y distintos insumos para la confección de calzado. También se encontraban en el mismo lugar talleres de confección y de reparación de calzado, los cuales recibían una alta demanda de trabajo.

En tal contexto, a principios de 1980, el barrio se enfrentó al fenómeno de las importaciones de productos desde China (tanto en su origen como producción), produciéndose un declive en la necesidad de fabricar calzados, ya que mucha la demanda se enfocaría en los productos de origen asiático, lo cual hizo entrar en crisis a la industria de calzado nacional y un número importante de locales cerraron sus puertas (Oficios

\footnotetext{
${ }^{1}$ Industrias tales como las textiles y carboníferas emplazadas en otras regiones del país (región de Biobío para este caso) se vieron afectadas por el proceso de desindustrialización y finalmente debieron cerrar. Dicha situación trajo consigo problemáticas asociadas a la incertidumbre laboral, pérdida de la memoria colectiva y de la cultura industrial. Frente a este panorama, en la actualidad, se está promoviendo la defensa y protección patrimonial, así como la conservación y reactivación de la memoria colectiva (Brito, 2018)
} 
Ulises Sepúlveda, Felipe Portales, Francisco Maturana: Experiencias de resistencia y agencia espacio-laboral de trabajadores de un barrio tradicional frente a cambios globalizadores: el Barrio Victoria, Santiago de Chile, Izquierdas, 49, julio 2020:1177-1197

Varios, 2014), reconfigurando así la geografía del comercio en la zona. En la actualidad, el barrio Victoria está constituido principalmente por distintos espacios laborales instaurados en tiempos y contextos diferentes. Junto con los espacios comerciales tradicionales, se han ido agregando con el pasar de los años nuevos tipos de espacios comerciales vinculados a la venta de calzado ya fabricado.

En este sentido, según Sandoval $(2012,153)$ la composición actual de locales comerciales en el barrio se debe a: "a) la transformación del giro de especialización, desde la venta de cueros al por mayor hacia la agregación de valor mediante los talleres artesanales del calzado y talabartería especializada y b) el desplazamiento geográfico de los comercios desde calle San Diego hacia calle Victoria en la búsqueda de mejores condiciones físicas y económicas para enfrentar la competencia externa”.

A partir de lo anterior y bajo la primicia de los aportes realizados en la geografía laboral, Buscar comprender las repercusiones de la reestructuración económica global en las experiencias de los trabajadores locatarios del barrio Victoria, toma relevancia, entendiendo que son los trabajadores el motor activo que puede reinventar un espacio que desea mantenerse dentro de las pautas actuales de producción y consumo. Es decir, desvelar las distintas formas de agencia laboral que los locatarios del barrio Victoria desarrollan a partir de prácticas de resiliencia, reelaboración o resistencia contextualizado en lo que Katz (2004) ha propuesto una triple categorización (“Las 3 R": resiliencia, reelaboración y resistencia).

Es necesario notar, que la resiliencia describe aquellos "pequeños actos de salir adelante, encontrar nuevas formas y maneras creativas de sobrevivir [...]" (Cumbers, Helms y Swanson, 2010), los cuales no desafían de manera relevante las relaciones sociales existentes. La reelaboración "difiere de la resiliencia al reflejar los intentos de las personas de crear espacios que puedan mejorar sus condiciones de existencia. En este sentido, implica un mayor nivel de conciencia de las condiciones subyacentes de la opresión, aunque al igual que la resiliencia no conduce necesariamente a la acción que desafía el poder hegemónico" (Cumbers, Helms y Swanson, 2010). Más bien, consistiría en un "intento de recalibrar las relaciones de poder y redistribuir los recursos" (Katz, 2004). Finalmente, las prácticas espaciales de resistencia consideradas en su máxima expresión- son más difíciles de evidenciar, ya que "implican desafíos directos a las relaciones sociales capitalistas, intentos de recuperar el control del tiempo de trabajo y su uso en las esferas de la producción y reproducción social" (Cumbers, Helms y Swanson, 2010). Este tipo de manifestación es escasa, puesto que, como explica Katz, requiere la invocación de una conciencia de oposición.

Por los anteriores, algunas interrogantes que surgen con la actual conformación espacio-laboral del barrio Victoria están asociadas a ¿qué obedece esa congregación de nuevos espacios comerciales? ¿Cómo se relacionan con los otros espacios de trabajo presentes en el barrio?

Las interrogantes planteadas buscan abordar el cómo de las interrogantes anteriormente expuestas y se estima que la manera de entregar una solución a ello es situar como objeto de estudio las acciones y las decisiones que los locatarios han tomado dentro de sus espacios de trabajo. Aquello, en definitiva, motiva la pregunta general de este trabajo: ¿cuáles son las formas de agencia espacio-laboral que construyen los locatarios en distintos tipos de locales del barrio Victoria?

Tal interrogante, permite poner en práctica la triple conceptualización de Katz (2004), para comprender las formas de agencia espacio laboral de los trabajadores del barrio Victoria. En efecto, tal aproximación 
Ulises Sepúlveda, Felipe Portales, Francisco Maturana: Experiencias de resistencia y agencia espacio-laboral de trabajadores de un barrio tradicional frente a cambios globalizadores: el Barrio Victoria, Santiago de Chile, Izquierdas, 49, julio 2020:1177-1197

fue de vital importancia para evidenciar cómo los diferentes espacios comerciales y productivos que conforman el barrio Victoria responden a diferentes prácticas agenciales iniciadas por sus locatarios 2 .

Por último, comenzar a validar y valorar estas prácticas agenciales desarrolladas por los trabajadores del barrio, puede ser una nueva opción para promover un complemento ${ }^{3}$ metodológico en el desarrollo de políticas públicas implementadas en distintas ciudades de Chile, que buscan revitalizar y dinamizar distintos barrios comerciales típicos.

Haciendo uso de metodologías cualitativas y con un enfoque socio-espacial para analizar barrios comerciales, es posible investigar cómo las prácticas laborales dispuestas por los trabajadores son la base de subsistencia y reproducción de estos espacios. Así en un primer momento, se identifica la lógica de trabajo tradicional del barrio y las dificultades laborales que conllevó la apertura económica del país. En un segundo paso se caracterizaron los distintos tipos de resiliencia y reelaboración implementada por los locatarios. Como tercer punto, se aborda una clasifican de las formas de agencia espacio-laboral por medio de prácticas y resiliencia y/o reelaboración y finalmente se analizaron las formas de agencia espacio-laboral de los locatarios del barrio Victoria.

\section{Metodología}

Para llevar a cabo esta investigación, se empleó una metodología en el contexto de la teoría fundamentada y la agencia espacio-laboral como concepto analítico. La selección de la muestra, fue basada en criterios. Tal tipo de muestreo, es una estrategia que busca el aseguramiento del rigor de la investigación por medio de la revisión y estudio de todos los casos que cumplen algún criterio predeterminado de importancia (Patton, 2015). Se tomaron en cuenta tres criterios. Las consideraciones de Tipo de trabajador y Temporalidad buscaron asegurar una profundización de las particularidades de las experiencias en los seis casos que se tomaron en cuenta para el análisis. Por otro lado, el criterio de Tipos de locales, respondió a una lógica de selección que pretende encontrar una mayor variación y diversidad en las lógicas de trabajo de los distintos locales en el contexto del barrio (ver cuadro 1). La distribución de los locales, se aprecia en la figura 1:

Cuadro 1. Criterios considerados para la selección de la muestra.

\begin{tabular}{|l|l|l|}
\hline $\mathrm{N}^{\circ}$ & Criterios & Características (Criterios 1 y 2) y Variables (Criterios 3 y 4) \\
\hline 1 & $\begin{array}{l}\text { Tipo de } \\
\text { trabajador }\end{array}$ & Locatario/as que arrienden o sean dueños de un local \\
\hline 2 & Temporalidad & $\begin{array}{l}\text { Los locatarios/as deben tener más de 20 años de experiencia en el barrio, } \\
\text { considerando también la experiencia previa de la familia (en caso de que el negocio } \\
\text { sea un legado familiar o de otro tipo) }\end{array}$ \\
\hline 3 & & 1. Local de venta de artículos confeccionados: LVAC \\
\hline
\end{tabular}

2 A modo de aclaración del sujeto de estudio, se entenderá por locatarios a todos los trabajadores que tienen un negocio o local a cargo dentro del barrio Victoria. No llegan a denominarse micro empresarios en el desarrollo de este trabajo, debido a ciertas condiciones y características que presentan. En primer lugar, son sujetos que administran un negocio, pero que a la vez desarrollan la mayoría de las labores de un trabajador asalariado dentro del espacio de trabajo; tareas tales como la fabricación, venta, contacto con clientes a distancia, mantención del orden y limpieza son comunes en ellos/as. Por otro lado, la propiedad de los negocios se ha obtenido por herencia familiar (algo bastante común en este tipo de espacios, en donde se desarrollan prácticas tradicionales que buscan mantenerse en el tiempo) o por medio del ahorro de muchos trabajadores dependientes que se apostaron por independizarse.

3 Refiere, de manera específica, a complementar metodológicamente el proyecto de "Fortalecimiento de barrios comerciales", implementado por el Gobierno de Chile y ejecutado por SERCOTEC, a causa de una falta de análisis espacial cualitativo en el desarrollo de la evaluación de resultados intermedios de dicho proyecto. (SERCOTEC, 2017) 


\begin{tabular}{|l|l|l|}
\hline \multirow{2}{*}{$\begin{array}{l}\text { Tipos de } \\
\text { locales }\end{array}$} & 2. Local de venta de materiales para confección: LVMC \\
\cline { 3 - 3 } & & 3. Taller de confección de artículos y productos: TCA \\
\cline { 2 - 3 } & 4. Taller y Local de venta de artículos confeccionados: TLVAC $\left.*^{*}\right)$ \\
\hline
\end{tabular}

Fuente: elaboración propia. $\left({ }^{*}\right)$ Los TLVAC corresponden a casos emergentes dentro de la investigación que son una derivación de los tipos de locales LVAC y TCA. Este tipo de caso no estaba contemplado durante la búsqueda. En el desarrollo de la teoría fundamentada (Charmaz, 2006) los datos empíricos emergentes ayudan a nutrir y otorgan consistencia a la conformación de la teoría.

Figura 1: Distribución de los distintos tipos de locales en el Barrio Victoria.

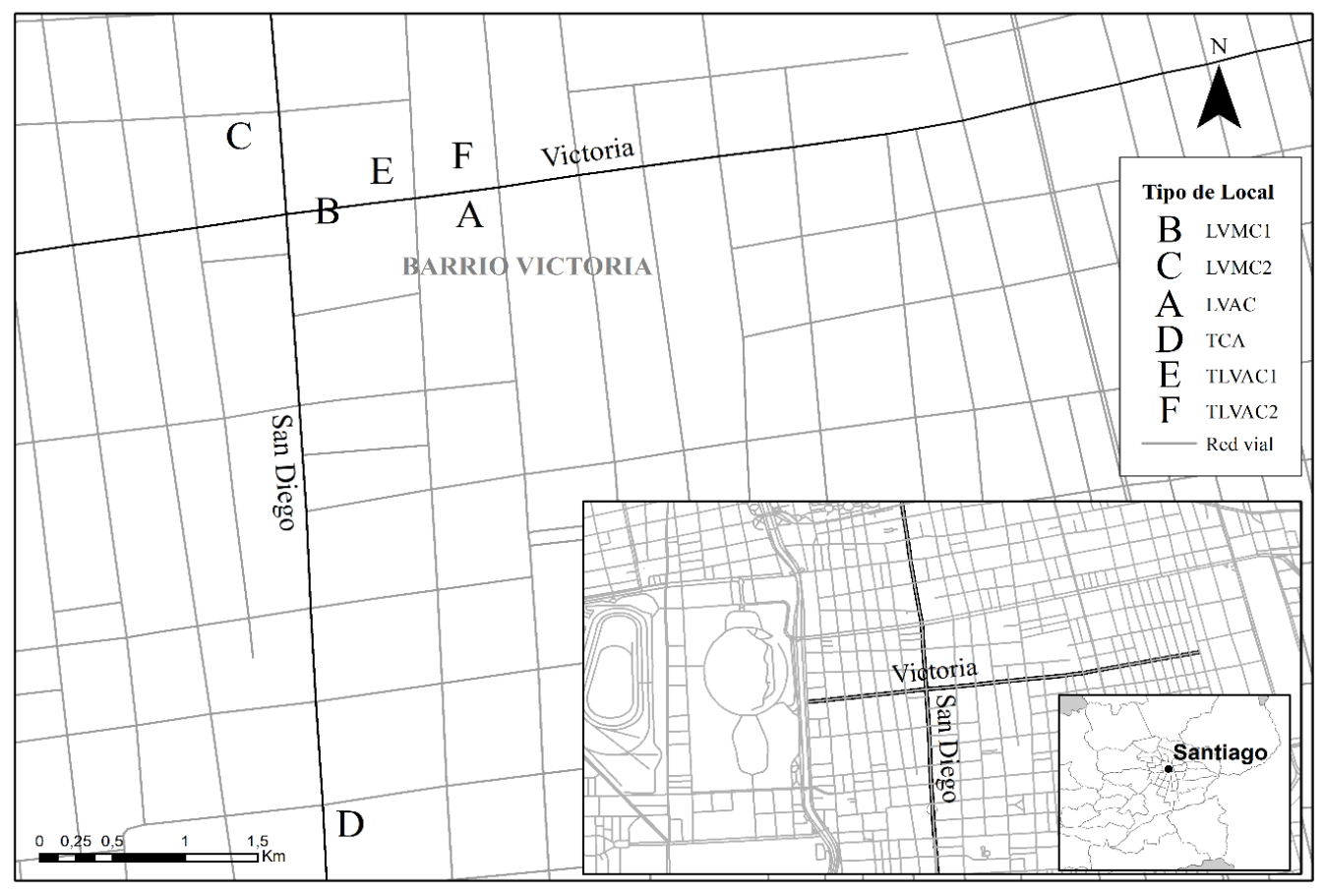

Fuente: elaboración propia con Capas base CEDEUS 2013, INE 2018 y BCN 2008 al 2010.

Cuadro 2. Casos seleccionados

\begin{tabular}{|l|c|}
\hline Casos seleccionados & Casos cantidad \\
\hline LVAC & 1 \\
\hline LVMC & 2 \\
\hline LVMC & 1 \\
\hline TLVAC & 2 \\
\hline
\end{tabular}

Fuente: elaboración propia.

Con el muestreo ya definido, se efectuaron entrevistas semiestructuradas inspiradas en las entrevistas topológicas (Sepúlveda, 2018), vinculando las experiencias espaciales de los sujetos. Estas entrevistas fueron guiadas por dos ejes temáticos que permiten relacionar a los sujetos con sus experiencias laborales: Historia y trabajados realizados en el barrio y Experiencia como locatario. El primero, tuvo por finalidad obtener información general de las dinámicas espacio-laborales dentro del barrio. Por otro lado, la segunda temática, buscó profundizar en las experiencias, acciones y decisiones del sujeto respecto de la administración de su negocio. Para ello, se disgregó la experiencia como locatario en tres sub tópicos: el negocio en sus inicios, distintos estados del negocio (periodos de crisis, regularidad, éxito) y las condiciones actuales y proyecciones. 
Ulises Sepúlveda, Felipe Portales, Francisco Maturana: Experiencias de resistencia y agencia espacio-laboral de trabajadores de un barrio tradicional frente a cambios globalizadores: el Barrio Victoria, Santiago de Chile, Izquierdas, 49, julio 2020:1177-1197

El proceso de recopilación de información, constó de dos instancias de reunión. En la primera, se desarrolló la entrevista con base en las temáticas descritas anteriormente y en la segunda, se realizó una entrevista que contempló interrogantes que surgieron producto de un primer análisis tentativo del material transcrito. Con tales dudas, se generó un esquema que se utilizó como herramienta de guía para una nueva recolección de datos.

Como se indicó, para llevar a cabo el análisis de las formas agenciales de los locatarios del barrio Victoria, se consideraron los principios de teoría fundamentada con un enfoque constructivista (Charmaz, 2006). Se utilizó el software de análisis de datos cualitativos Atlas.ti, en donde se realizó una codificación línea por línea, proceso a través del cual se asigna un código a cada renglón de datos. De esta codificación inicial o abierta, surgen los primeros conceptos con énfasis en los datos textuales. Los códigos iniciales son provisionales, comparativos y se basan en los datos. Son provisionales, puesto que pretenden permanecer abiertos a otras posibilidades analíticas y crear códigos que se ajusten mejor a los datos que se tienen (Charmaz, 2006).

Estos códigos iniciales, se enfocan en las decisiones y acciones tomadas por locatarios en distintas circunstancias laborales, es decir, en periodos de crisis y dificultades, pero también en momentos de transición y estabilidad. Así, estos códigos buscaron englobar el campo de acción de estos locatarios en su espacio. Los códigos emergentes, de primer orden, entregaron forma y sentido a las categorías conceptuales denominadas prácticas de resiliencia y/o reelaboración.

Los resultados de segundo orden, corresponden al proceso de codificación focalizada. Esta, consiste en utilizar los códigos anteriores más significativos y/o más frecuentes y ver si éstos funcionan al aplicarlos a una gran cantidad de datos (Charmaz, 2006). Este proceso, permitió reconocer y analizar las distintas relaciones e interacciones entre los distintos espacios de trabajo.

Con los procesos generales de análisis, se logró elaborar un modelo emergente, que permitió interpretar los distintos mecanismos y cambios que los locatarios han realizado en sus espacios de trabajo y cómo esto influye en las relaciones de los mismos. Además, estas últimas, evidencian las deficiencias en los procesos de producción y reproducción dentro del barrio Victoria, así como también las distintas formas de subsistencia.

\section{Resultados}

\section{Cualidades laborales de los sujetos: el trabajo independiente y la autonomía}

La particularidad de los locatarios del barrio Victoria, es que corresponden a una tipología de trabajadores que tiene cierto grado de autonomía. Esta, se relaciona con la libertad con la que cuentan a la hora de administrar el negocio, es decir, tienen la potestad de decidir qué se fabricará, qué insumos y materiales se compraran y a qué tipo de clientes se busca atraer (por mencionar algunos aspectos).

Además, tal condición, si bien permite tomar las riendas del negocio, también conlleva una carga de decisiones y labores simultáneas de las que se deben hacer cargo. Es de primera necesidad, contar con un amplio conocimiento de productos y materiales que se venden y tener una clientela que consuma de forma periódica, puesto que son el sustento del negocio. Además, es necesario contar con una red de contactos relacionados al abastecimiento de materiales y productos. En última instancia, deben buscar formas de difundir y publicitar su trabajo. 


\section{Lógica espacial de trabajo precedente a la apertura económica del país}

Según los tipos de espacios laborales identificados en el barrio y sus lógicas de trabajo (ver figura 2), se pudo observar que existía una concentración de espacios de abastecimiento que tenían una alta demanda de materiales para la confección de calzado. Esta situación, suponía el manejo de un stock cíclico que corresponde a la mantención de gran cantidad de mercadería por parte de los locatarios, debido a que tenían la certeza de la pronta venta de esos materiales. Los altos requerimientos de suministro, también se extrapolaban a las curtiembres, que eran los principales espacios de abastecimiento en lo que refiere al cuero. Otra particularidad que tenía el ciclo productivo del calzado, es que estaba (y está aún) supeditado a un aumento de ventas por temporada, ya que en las distintas estaciones del año se concentran las solicitudes de fabricación y ventas en ciertos tipos de calzado.

Por otro lado, al ser un barrio especializado en suministros de materiales para la fabricación y reparación de calzados, la clientela era bastante homogénea: "Esta calle comenzaba a funcionar a las siete de la mañana, las suelerías abrían y todos los zapateros venían a comprar materiales, los zapateros remendones, los fabricantes" (TLVAC2).

Figura 2. Lógica de abastecimiento en el barrio Victoria.

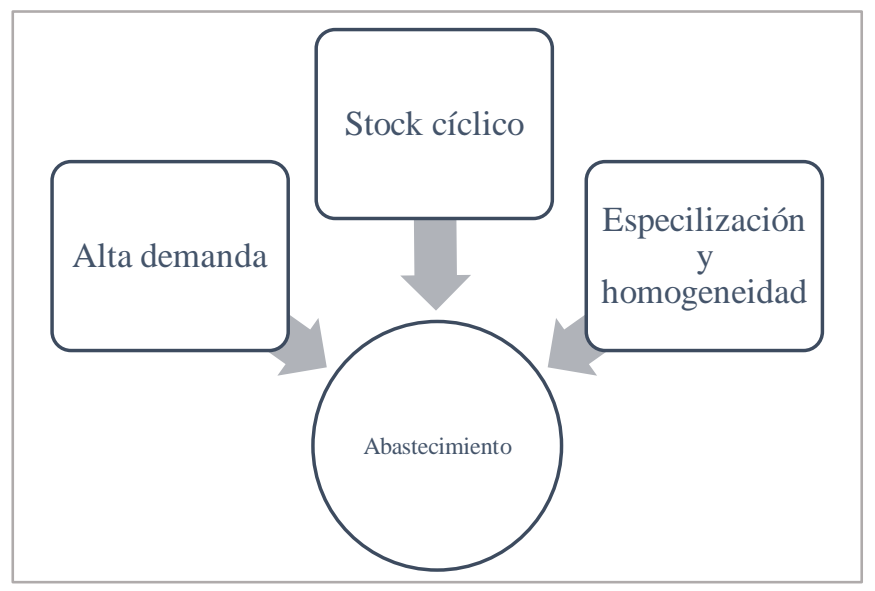

Fuente: elaboración propia.

Figura 3. Interacción entre los espacios que componen la relación productiva del calzado.

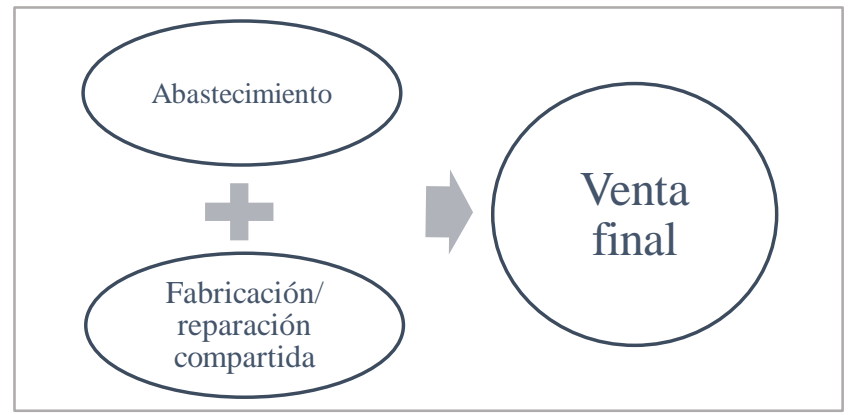

Fuente: elaboración propia.

La interacción entre los espacios de abastecimiento, espacios de reparación/fabricación y espacios de venta final, no se concentraba en el mismo barrio Victoria. El barrio, tradicionalmente funcionó como 
Ulises Sepúlveda, Felipe Portales, Francisco Maturana: Experiencias de resistencia y agencia espacio-laboral de trabajadores de un barrio tradicional frente a cambios globalizadores: el Barrio Victoria, Santiago de Chile, Izquierdas, 49, julio 2020:1177-1197

espacio de abastecimiento y provisión. La reparación, la fabricación y los espacios de venta final, se encontraban al exterior del lugar. Con el pasar del tiempo, se comenzaron a incorporar talleres y fábricas dentro del barrio, pero este nunca fue concebido como un espacio comercial de venta final en donde se congregarán tiendas que ofrecen productos que ya pasaron por toda la cadena productiva como se aprecia en la figura 3 (ver página anterior).

\section{Las dificultades que supone la importación de calzado en un barrio especializado en dicho producto}

La baja considerable en la demanda de abastecimiento, reparación y fabricación, es el principal problema que surge con la importación de calzado de origen asiático. Emergen nuevos espacios comerciales dentro de la ciudad de Santiago, que comienzan a agrupar la venta de distintos tipos de producto importado, los cuales atraen la mayor masa de consumidores de calzado. Las transformaciones más significativas corresponden a la desaparición masiva de espacios comerciales de abastecimiento dentro del barrio: “...había muchas suelerías, alrededor de 30, 40 suelerías que eran las que vendían todos los productos para hacer calzados, y se hacían pocas. Hoy, sin embargo, quedan cinco, seis, diez tal vez, con suerte" (LVMC2). Asimismo, la migración de mano de obra especializada a otros sectores laborales, supuso otra alteración en la configuración espacio-comercial del barrio.

También se encontraron dificultades inherentes del barrio que limitan la agencia laboral por parte de los locatarios. Una, refiere a la propiedad de los locales. Los propietarios de estos locales -que en sus inicios fueron espacios residenciales- son unos pocos, lo que se traduce en un monopolio del arriendo de estos locales, en donde prácticamente todos los locatarios que tienen su negocio deben arrendar. Esta condición, limita la agencia laboral, sobre todo por el costo económico que comporta el arriendo: "Mi amigo que te presenté ese día paga 700 lucas [mil pesos] ${ }^{4}$ de arriendo y ahí a la vuelta tiene un taller que paga 360. Tiene un millón de pesos de arriendo, y no da po’ [no alcanza]" (TCA).

Además, se reconoce una problemática socio-cultural propia de los trabajadores antiguos del barrio, que se relaciona con el bajo nivel de educación y preparación que estos tenían para manejar el negocio. Esta carencia, supuso -en algunos casos- pésimos negocios, desperdicio y mal uso del capital y, sobre todo, una respuesta deficiente ante al fenómeno de importación de calzado de origen asiático: "es una cuestión cultural y de educación del mismo zapatero, el zapatero viene muy de abajo, le ha costado salir y proyectarse más adelante" (TLVAC1).

\section{Un modelo de análisis espacial emergente}

El modelo emergente, se elaboró por medio de los procedimientos básicos que indica la teoría fundamentada para la etapa de codificación de la información obtenida de los casos estudiados. En la primera etapa de codificación inicial o abierta, surgieron códigos que daban cuenta de las prácticas y decisiones que los locatarios han llevado a cabo para administrar su negocio. Todas estas acciones, se englobaron en lo que se denominó "lógica de trabajo", la cual viene a explicar la forma de realizar distintas tareas y aspectos dentro de cada espacio laboral. A esta lógica de trabajo, se le da una forma y sentido por medio de los códigos que indican y fundamentan los distintos tipos de productos/servicios que el local ofrece, cómo se maneja el stock, los clientes en los cuales se enfoca y los proveedores con los que se relaciona (ver figura 4). Estas distintas variables, configuran diferentes lógicas de trabajo que difieren en cada tipo de local analizado.

${ }^{4}$ Considerar el equivalente a algo de 900 euros, según tipo de cambio. 
Figura 4. Codificación inicial y categorías emergentes.

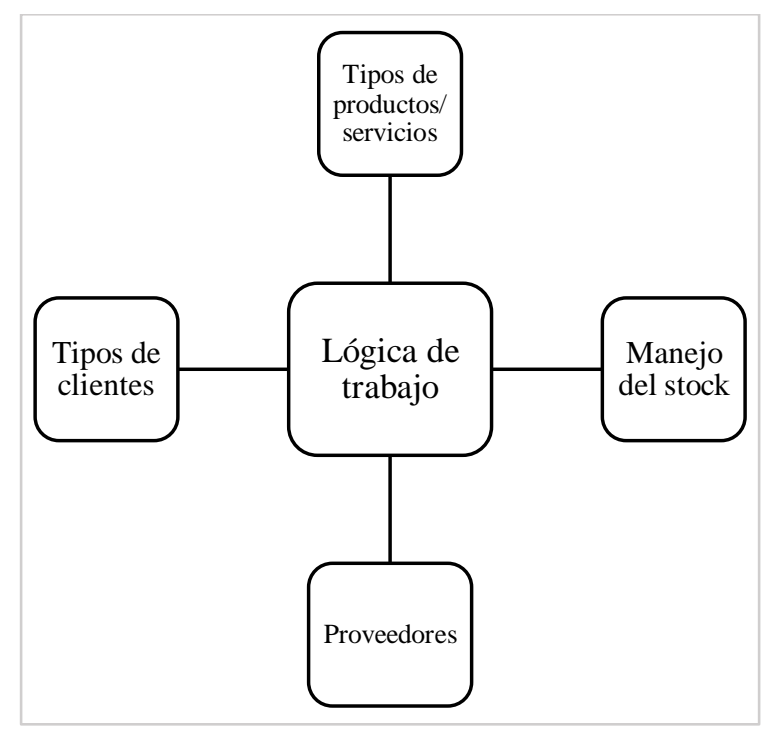

Fuente: elaboración propia.

Luego, estas lógicas de trabajo se asociaron a los distintos niveles de acción que los locatarios han implementado. Estos niveles de acción se explican por medio de las categorías de prácticas de resiliencia y reelaboración inspiradas en el trabajo de Katz (2004).

Figura 5. Codificación focalizada. Análisis relacional de los distintos espacios.

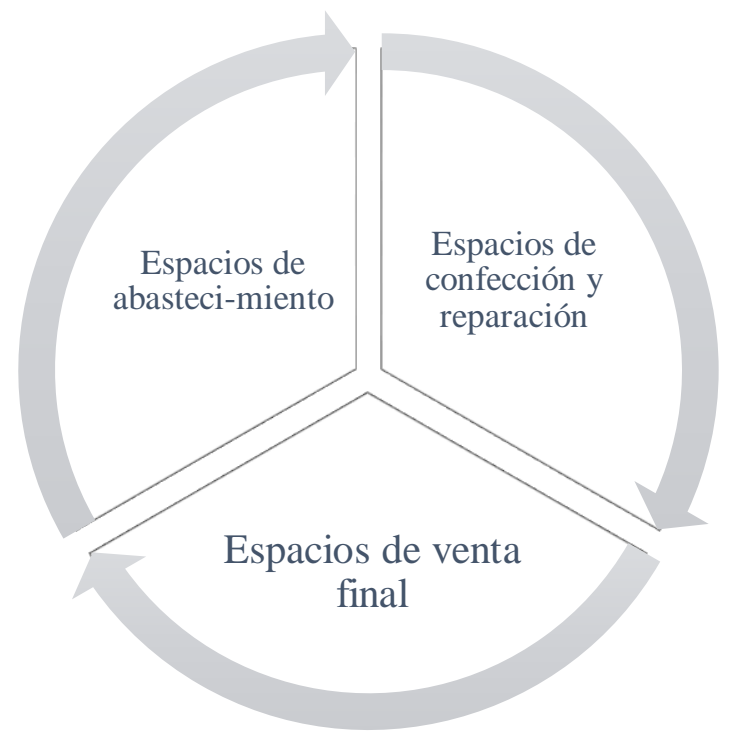

Fuente: elaboración propia.

Las distintas formas agenciales, se fundamentan a través de las prácticas de resiliencia y/o reelaboración que son las categorías de primer orden. Estas, conceptualizan los distintos métodos que los locatarios 
han utilizado para "gestionar el negocio", lo que permite la subsistencia de estos tipos de espacios comerciales.

En la segunda fase de codificación, se realizó un análisis relacional de los conceptos emergentes, es decir, se compararon y relacionaron las diferentes lógicas de trabajo que dan cuenta de distintas prácticas de resiliencia y/o reelaboración en cada caso. Dicho de otra forma, en ese paso se determinaron las relaciones espacio-laborales que han surgido de estas organizaciones de la economía de los distintos locales de trabajo. Estas diversas relaciones entre espacios al interior del barrio Victoria, generan configuraciones denominadas micro geografías de producción y reproducción laboral que permiten la subsistencia de los locatarios por medio de las formas de agencia espacio-laboral que estos han implementado (ver figura 6).

Tales micro geografías, identifican tres espacios de trabajo principales, los de abastecimiento, confección/ reparación y de venta final (ver figura 5). Por medio de estas relaciones, se logró identificar las principales propiedades de cada espacio, las funciones que cumple cada uno en relación con los otros, logrando comprender, además, el alcance escalar de estas relaciones, es decir, si existen relaciones de abastecimiento, producción y comercio que escapan al barrio Victoria.

Figura 6. Modelo de análisis

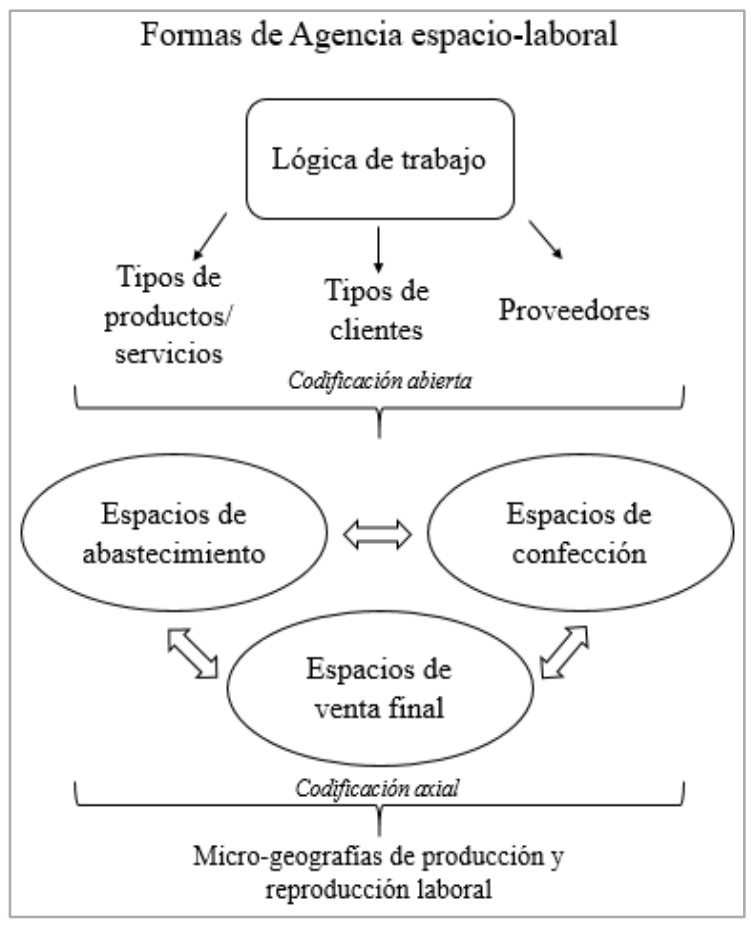

Fuente: elaboración propia.

En definitiva, los resultados que entregan estas micro-geografias de producción y reproducción laboral permiten explicar, desde los locatarios, cómo el barrio Victoria sigue subsistiendo y se adapta a lógicas globales a través de las diferentes relaciones que se configuran entre los distintos espacios laborales presentes en él (ver figura 6). 


\section{Resultados de primer orden: cambios en las lógicas de trabajo mediadas por prácticas de resiliencia y/o reelaboración}

Estos resultados, expresan los cambios implementados en las lógicas de trabajo de cada espacio laboral. Mencionar también, que las prácticas de resiliencia (de menor nivel de acción), se concentran en los espacios de abastecimiento y están influenciadas principalmente por las prácticas de reelaboración que han impuesto los locatarios de LVAC, TLVAC y TAC (locales enfocados en la confección/ reparación y venta final). A continuación, se muestran los principales cambios implementados en cada tipo de espacio de trabajo.

\section{Resiliencia: adaptación en el abastecimiento}

Figura 7. Adaptación en el abastecimiento y códigos asociados.

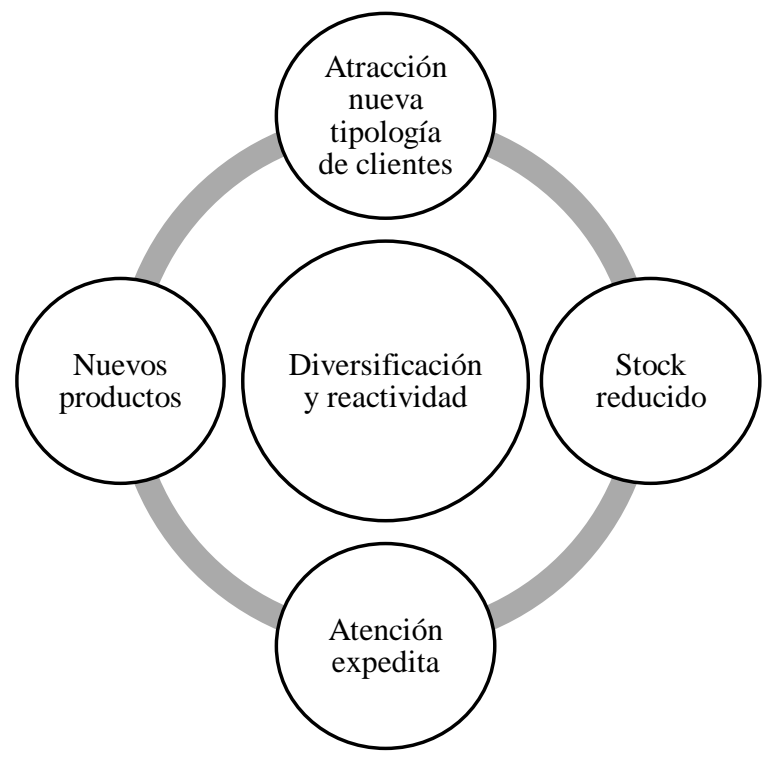

Fuente: elaboración propia.

Como se aprecia en la figura 7, la adaptación en el abastecimiento supone dos prácticas de resiliencia fundamentales: la diversificación en la oferta de productos (materias primas para la confección y reparación), es decir, se comienzan a ofrecer nuevos productos que ya no se relacionan con el segmento del calzado dentro del rubro del cuero, sino que atienden las necesidades y demandas de otra tipología de clientes que ya no son sólo "zapateros".

Por otro lado, el manejo de un stock reactivo, implica el abastecimiento en bajas cantidades de mercadería por temporada (a diferencia del manejo del stock cíclico). El abastecimiento, por tanto, ya no responde a una periodicidad y frecuencias marcadas, por el contrario, se adapta a las nuevas dinámicas de los trabajos a pedido y de diversificación que no obedecen a pautas definidas de fabricación y venta: "Hoy día se va comprando a medida que tú vas necesitando y las cantidades y volúmenes que tú tienes en este momento de bodega son mínimas, ósea, con suerte, tal vez, para diez días, no más. Los proveedores se han ido también adaptando al mismo sistema. Ellos están fabricando, fabrican menos, pero son más rápidos en la entrega, entonces, nos ajustamos todos al mismo sistema” (LVMC2). 


\section{Reelaboración: segmentación del rubro}

Como expresa la figura 8, estas prácticas, corresponden a una segmentación del rubro, que responden a lógicas de trabajo a pedido y a la tercerización en la confección. Esto quiere decir, que tanto fabricantes como vendedores están apostando por la agregación de valor, ofreciendo un servicio más especializado y en el menor tiempo posible. Este trabajo especializado (o a pedido), pone énfasis en la relación precio/ calidad de los productos y servicios que se ofrecen y se acopla a una forma de trabajar que requiere un escaso personal de trabajo (el cual debe ser especialista en las laborales que realiza). Así, esta forma, responde a una estrategia que busca sopesar la ausencia de pick de ventas, por tanto, se trabaja de forma continua con proyectos que implican un mayor tiempo y valor agregado.

En segundo lugar, la tercerización apunta a la fabricación externa con el fin de responder a la incertidumbre laboral que supone la baja demanda de trabajo. La tercerización, también otorga oportunidades a pequeños fabricantes externos al barrio, que no tienen la posibilidad de tener su propio espacio, generando así una cadena que busca mantener la producción de baja escala.

Este cambio en la lógica de trabajo dentro del rubro, conlleva un desafío para los espacios de abastecimiento (principalmente curtiembres y suelerías) que se deben adaptar a los requerimientos de los espacios de fabricación/reparación y de venta final.

Figura 8. Segmentación del rubro y códigos asociados.

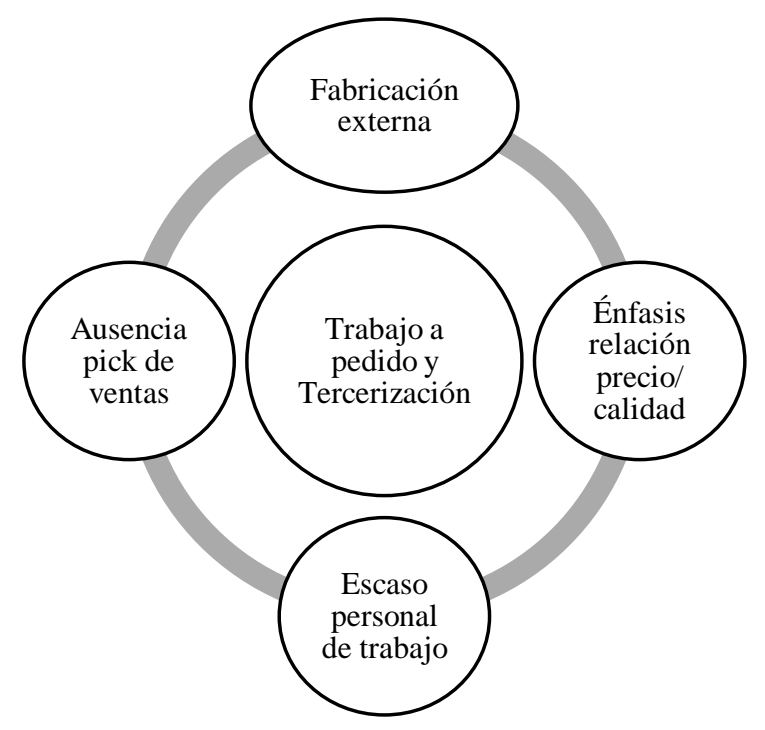

Fuente: elaboración propia.

\section{Reelaboración: articulación de espacios}

Como última forma de reelaboración, se encuentra la articulación de espacios de fabricación/ reparación y prestación con los de venta final que se ve expresada en la figura 9. Esta articulación, responde a una lógica de complemento en la oferta de productos y servicios, que busca sopesar la baja demanda, ampliando así la oferta de estos locales. La finalidad, atraer la mayor cantidad de clientes posibles utilizando dos "formatos de trabajo", uno que tiene que ver con el trabajo a pedido, en donde estos espacios solicitan -a los espacios de abastecimiento- materiales a medida que fabrican. 
Figura 9. Articulación de espacios y códigos asociados.

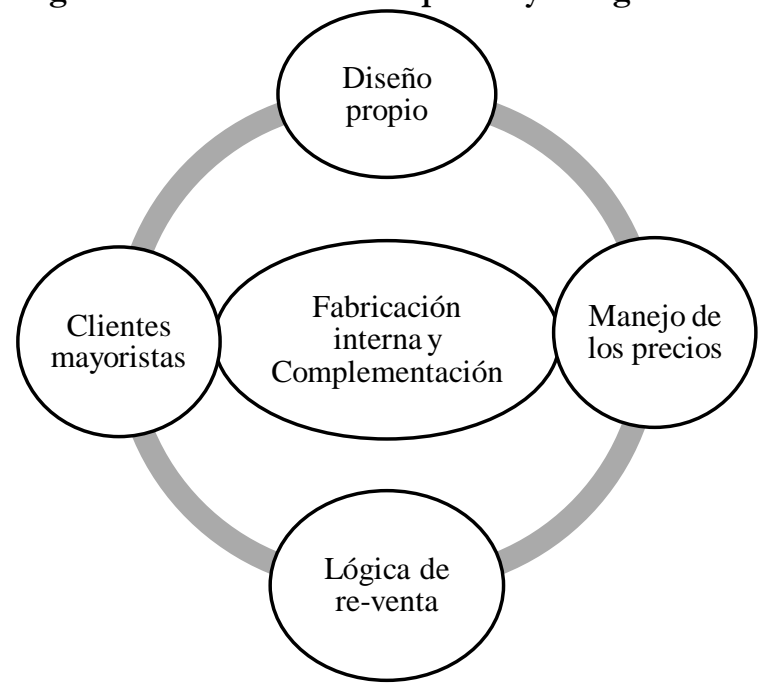

Fuente: elaboración propia.

Figura 10. Prácticas de resiliencia y/o reelaboración y sus relaciones.

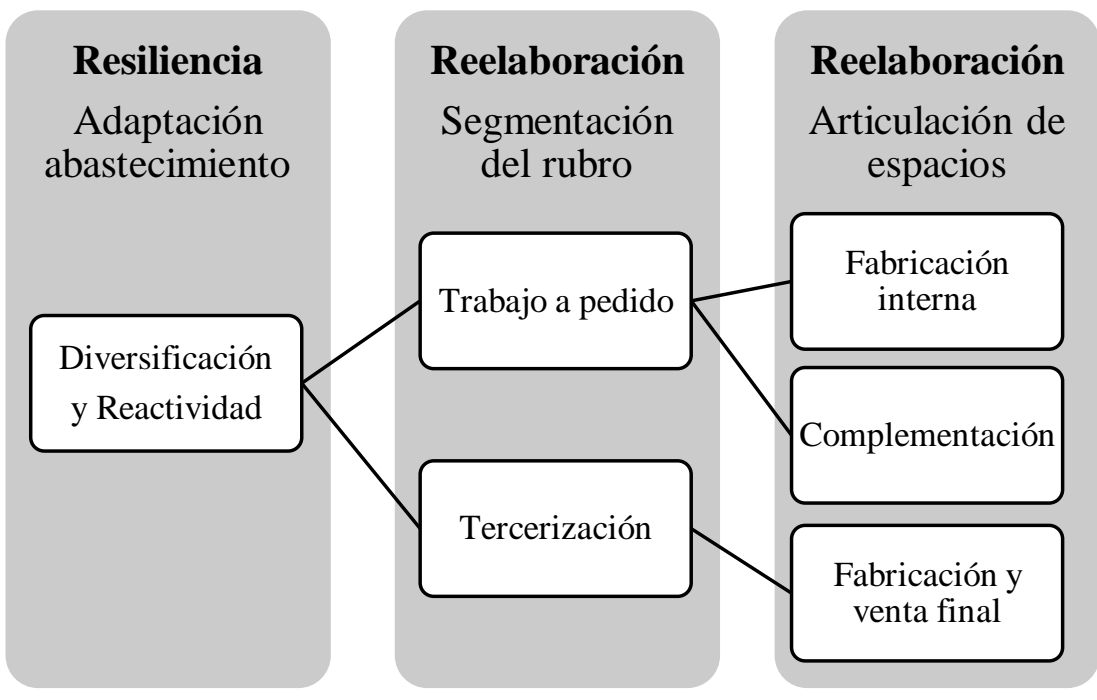

Fuente: elaboración propia.

Un segundo elemento, tiene que ver con la incorporación de la tienda (venta de productos ya confeccionados), en donde se demandan materiales para la confección de manera continua, ya que se mantiene un stock mínimo de productos para la venta directa. En resumidas cuentas, con el taller se trabaja a pedido y, en consecuencia, la venta no se realiza de manera instantánea. En cambio, en la tienda, se requiere de un trabajo anterior para que el producto ya esté fabricado y listo para comercializarse. Esta lógica complementaria -que genera la articulación de espacios- expresa otra dualidad: la fabricación interna para la re-venta y tercerización de la fabricación. 
La fabricación interna, permite a los locatarios reducir costos que permiten realizar ventas mayoristas que se traducen en re-ventas: "nosotros también vendemos... somos mayoristas, le vendemos a varias chicas que venden por Instagram el calzado, obviamente ellas doblan los precios" (LVAC). La lógica de reventa, que se da por medio de la fabricación interna, también entrega la facilidad de poder diseñar los productos que se ofrecen y manejar los precios en relación a la oferta y demanda. La tercerización del trabajo, minimiza los riesgos que supone la baja demanda de productos y servicios: "Por un tema logístico de espacio y pega, porque al final hoy día no puedo contratar gente pa echarlos mañana. Sin embargo, ellos tienen sus talleres y yo les doy pega cuando la necesito" (TLVAC2).

\section{Resultados de segundo orden: micro geografías de producción y reproducción laboral}

Figura 11. Micro geografías de producción y reproducción laboral.

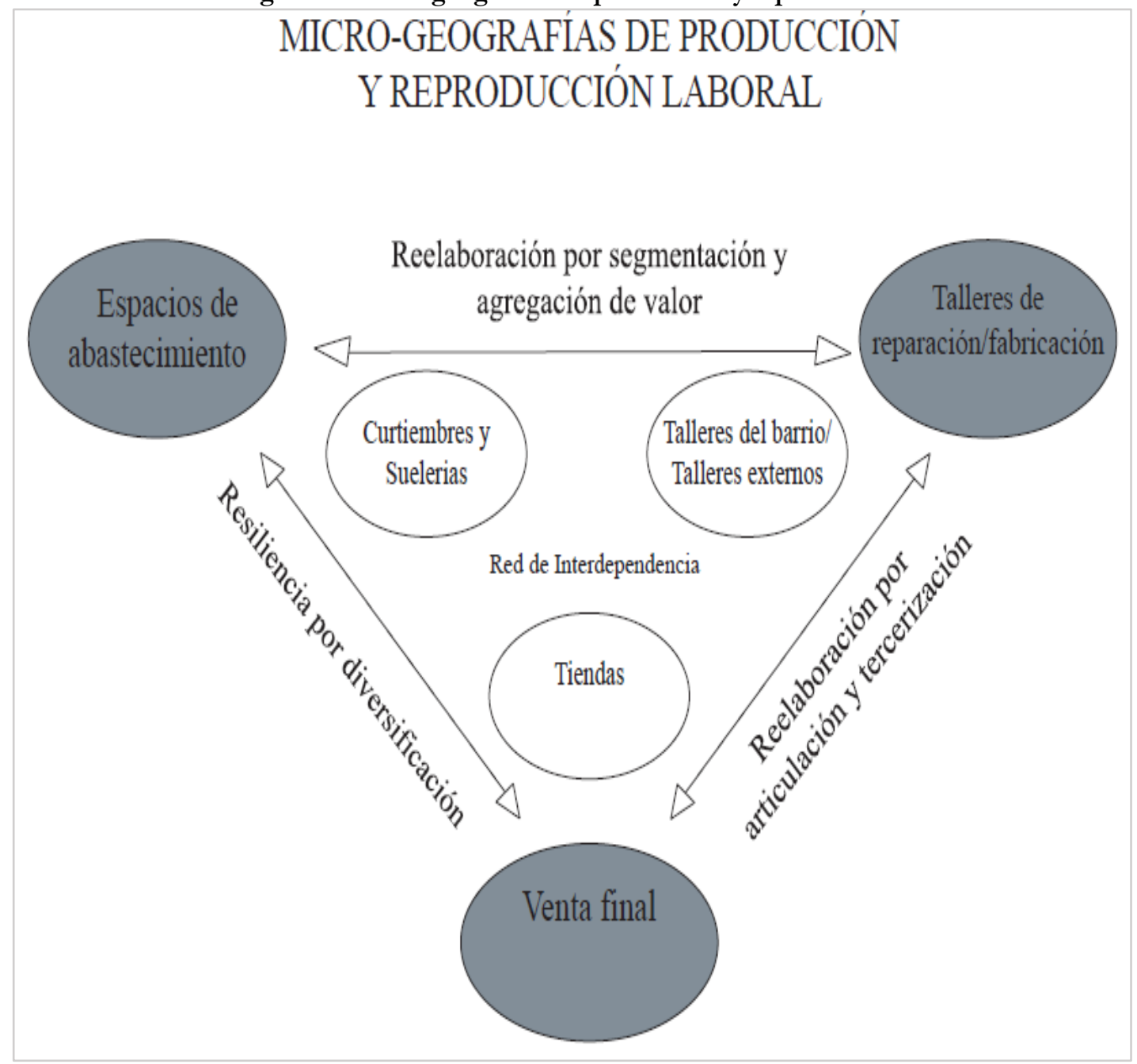

Fuente: elaboración propia.

La incorporación de los espacios de venta final, supuso una reconfiguración que complejiza las relaciones entre todos los espacios laborales presentes en el barrio, y las de estos con los espacios externos a él (ver figura 11). Esto, generó nuevas dinámicas en las formas de fabricación y abastecimiento. A continuación, 
Ulises Sepúlveda, Felipe Portales, Francisco Maturana: Experiencias de resistencia y agencia espacio-laboral de trabajadores de un barrio tradicional frente a cambios globalizadores: el Barrio Victoria, Santiago de Chile, Izquierdas, 49, julio 2020:1177-1197

se explicará cómo las prácticas de reelaboración y resiliencia -efectuadas en un espacio- han incidido en los demás. Son tres configuraciones que emergen (ver figura 12):

Figura 12. Tres configuraciones relacionales entre los distintos espacios presentes en el barrio. Fuente: elaboración propia.
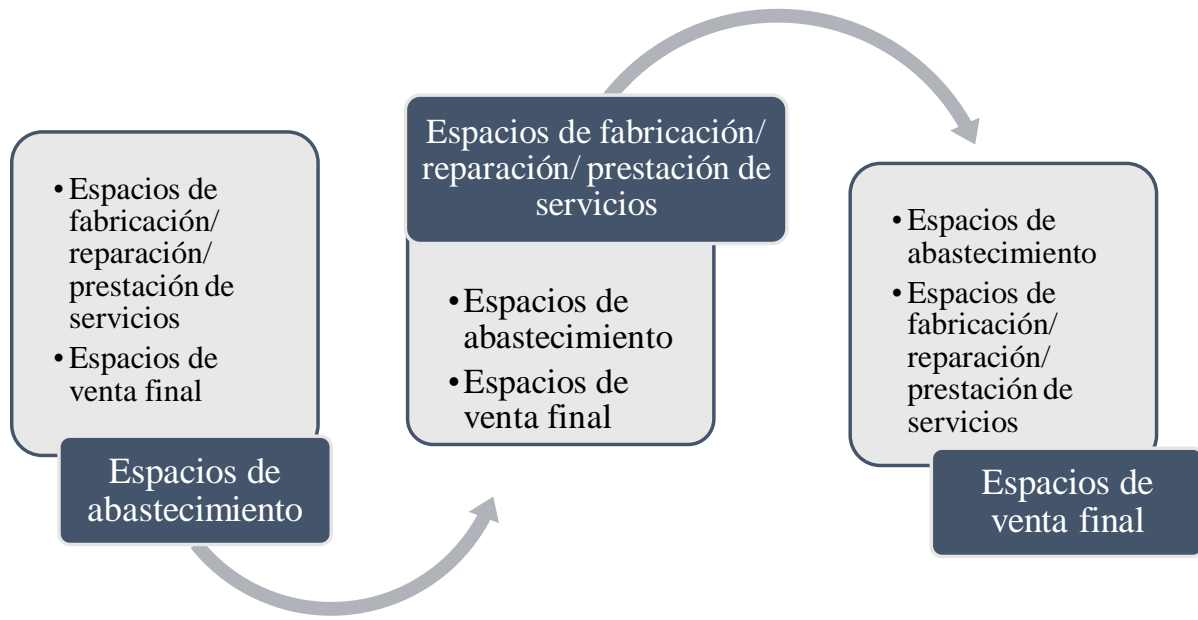

La primera configuración, obedece a los cambios realizados desde los espacios de abastecimiento. En una cadena productiva, la provisión está dada por una demanda que requiere de ello, por tanto, como se mencionó anteriormente, estos espacios de suministro están supeditados a las agencias que se efectúan, en este caso, en los otros dos espacios. Esto último, quiere decir que estos espacios no son centrales dentro de la configuración de nuevas geografías dentro del barrio. El papel que juegan estos espacios, es el de atender las necesidades en la fabricación y venta final. Estas demandas desde los otros lugares, las han logrado resolver por medio de la llamada resiliencia por diversificación y reactividad.

La segunda configuración, se enfoca en los espacios de fabricación/reparación/ prestación de servicios, los cuales, a nivel relacional, demandan materiales a los espacios de abastecimiento y funcionan como lugares de suministro para los espacios de venta final y del trabajo a pedido. Con frecuencia, son los clientes que proveen materiales necesarios para la confección de los productos solicitados.

La tercera configuración relacional, se orienta hacia los espacios de venta final y presenta dos tipos de micro-geografías que se vinculan con a) la tercerización y b) la producción interna:

a) La primera micro-geografía se relaciona con la venta directa. Los productos que se ofrecen aquí, provienen muchas veces de una producción tercerizada (Reelaboración por tercerización). Es decir, los locatarios compran los insumos y materiales para la producción y la confección la derivan a talleres externos. La tercerización, responde en muchos casos, a una producción de menor tamaño que busca complementar espacios que se han dedicado a la fabricación y/o a la prestación de servicios.

b) La segunda, responde a la lógica de re-venta. En este caso, los espacios de venta final situados en el barrio funcionan como espacios de abastecimiento de venta final para los clientes que compran productos en grandes cantidades. Esta posibilidad -de ser un espacio de abastecimiento para la venta final- se ha podido lograr por la denominada reelaboración por articulación de espacios, en donde estos, tienen la capacidad de funcionar como un lugar de abastecimiento para otros sectores de 
Ulises Sepúlveda, Felipe Portales, Francisco Maturana: Experiencias de resistencia y agencia espacio-laboral de trabajadores de un barrio tradicional frente a cambios globalizadores: el Barrio Victoria, Santiago de Chile, Izquierdas, 49, julio 2020:1177-1197

venta (ya sea externos al barrio, o internos), por la capacidad de producción que les otorga el tener un espacio de fabricación propio.

Estas micro geografías, relevan la capacidad de subsistencia que han tenido estos espacios. Es decir, la adaptación de las suelerias a los nuevos requerimientos de fabricantes y vendedores. Los fabricantes, con nuevas formas de confeccionar que atraen a nuevos tipos de clientes y que integran, junto con su producción, la comercialización de sus productos y otros de origen externos al barrio. Por su parte, los comerciantes de productos confeccionados (los nuevos actores), que intentan revitalizar el barrio integrando la venta final, lo que ha posibilitado un giro comercial que atrae una mayor diversidad de clientes.

\section{Conclusiones}

El uso de la agencia espacio-laboral como concepto base de análisis, en conjunto con las prácticas de resiliencia y reelaboración, entregó resultados que evidencian nuevas relaciones de producción y reproducción que escapan a la espacialidad del barrio Victoria en una ciudad de características globales como lo es Santiago en la actualidad. Esto indica, que las formas de interacción socio espacial dentro del barrio, se han modificado para conformar nuevas geografías económicas que buscan atender renovadas necesidades tanto de los espacios exteriores al barrio Victoria como dentro de él. Un ejemplo de aquello, es la búsqueda de la diversificación de los productos y la exclusividad en la fabricación. Esta exclusividad en el diseño, más el aporte de la calidad que supone el cuero, ha promovido nuevas demandas desde espacios comerciales exteriores que buscan comprar estos productos a un bajo costo, para luego comercializarlos a un precio superior en otros lugares de venta.

Las prácticas de resiliencia y reelaboración implementadas por los locatarios, también dan cuenta de acciones de complementariedad en la cadena productiva, buscando la subsistencia y reproducción de este barrio, por medio de la retroalimentación entre espacios de fabricación, reparación y venta final. La agencia laboral, resulta ser de ayuda para comprender cómo a partir de las acciones y decisiones que toman distintos tipos de trabajadores en diferentes espacios laborales (en este caso, de producción y comercio), conforman nuevas relaciones productivas, en donde espacios que cumplían una función dentro de una red de trabajo, pasan a complementar esa función o radicalmente modificar esa labor dentro de la configuración espacial.

Estos cambios de configuración dentro de las geografías productivas del barrio, pueden dar cuenta de cuáles fueron las causas de esos cambios y a qué se deben. En este sentido, el uso de la triple conceptualización de Katz, permitió entregar una mayor solidez al análisis de las agencias espaciolaborales. Así, tanto las prácticas de resiliencia como las de reelaboración, son disgregaciones del uso de distintas formas de agencia que permitieron profundizar diferentes aspectos relacionados a las lógicas de trabajo de los sujetos estudiados.

Es probable, que las micro geografías de producción y reproducción expuestas muten en el futuro, sobre todo con la conformación de nuevas relaciones espaciales que supone la expansión y segmentación dentro del rubro del cuero y la diversificación de productos que ha resultado una estrategia eficaz para atraer nuevos clientes.

Por ejemplo, otros tipos de espacios comerciales que comienzan a interactuar cada vez más con el barrio Victoria son las redes sociales y sus plataformas de venta online. Estudiar este fenómeno de venta por internet y entender cómo se relacionan con espacios comerciales tradicionales en barrios típicos de Santiago, emerge como otra problemática posible de estudiar. 
Ulises Sepúlveda, Felipe Portales, Francisco Maturana: Experiencias de resistencia y agencia espacio-laboral de trabajadores de un barrio tradicional frente a cambios globalizadores: el Barrio Victoria, Santiago de Chile, Izquierdas, 49, julio 2020:1177-1197

También, debería considerarse el nuevo contexto socio-cultural de valorización paulatina de la calidad de los productos, y de lo artesanal y local frente a los productos importados.

Para trabajos posteriores que se dediquen a la investigación de estas prácticas (agenciales), sería interesante integrar algunos cuestionamientos que han surgido en torno al desarrollo de la agencia en la geografía laboral. Una de las principales incertidumbres, es si la agencia laboral considera las intenciones o las consecuencias de las prácticas (Hauge y Fold, 2016). El objetivo de esta nueva interrogante, es integrar en el enfoque analítico esta dualidad: intenciones/consecuencias.

Las intenciones, serían cruciales para para comprender las lógicas de las acciones y están inseparablemente conectadas con las condiciones socioeconómicas de los hogares de los trabajadores (Hauge y Fold, 2016). En ese sentido, se podría avanzar en la comprensión de la agencia considerando las condiciones e incidencias que efectúan los espacios de reproducción en los trabajadores.

Las consecuencias, serían los resultados esperados y no deseados de las prácticas y pueden coincidir o no con las intenciones que, por así decirlo, crearon (Jones y Murphy, 2010). El quiebre que se produce entre las intenciones y las consecuencias puede resultar revelador. Cuando los resultados no coincidan con las intenciones que se proponen, se estarían revelando las brechas y necesidades socio-económicas que condicionan el éxito o logro de estas intenciones iniciales.

Finalmente, la integración de los avances de la geografía laboral en el desarrollo de políticas públicas podría ser relevante, en cuanto a promover un análisis más integral de las problemáticas que se quieran solucionar. En el caso del proyecto de mejoramiento de barrios típicos comerciales ejecutado por SERCOTEC, sería un avance complementar los diagnósticos cuantitativos y resultados descriptivos por medio de la incorporación de un método que identifique los espacios de trabajo presentes en cada barrio y con un análisis espacial a partir de los trabajadores que lo conforman, logrando así, identificar los espacios de trabajo que no se han considerado dentro de la implementación de políticas públicas que benefician a barrios tradicionales de distintas ciudades de Chile y por tanto contribuir -desde una óptica espacial y socio-económica- la conservación de los modos de vida tradicionales dentro de la ciudad, los cuales cada vez se ven más influenciados por las nuevas dinámicas globales.

\section{Referencias}

\section{Fuentes impresas}

Brito, Alejandra, "Memoria colectiva y construcción de territorio: auge y despojo de una cultura industrial. Los casos de la fábrica textil Bellavista-Tomé y la carbonífera Schwager en Coronel (1970-2007)", Izquierdas, 42, 2018, 1-29.

Carmagnani, Marcelo, Desarrollo industrial y subdesarrollo económico del caso cbileno (1860-1920), Santiago, DIBAM, 1998.

Carswell, Grace \& De Neve, Geert, "Labouring for global markets: Conceptualising labour agency in global production networks", Geoforum, 44, 2013, 62-70.

Charmaz, Kathy, Constructing Grounded Theory: A practical buide through qualitative analysis, SAGE Publications, 2006. 
Coe, Neil \& Hess, Martin, "Global production networks, labour and development. Geoforum”, 44, 2013, 4-9.

Cumbers, Andrew, Helms, Gesa \& Swanson, Kate, "Class, Agency and Resistance in the Old Industrial City", Antipode, 42, 2010, 46-73.

De Mattos, Carlos, "Santiago de Chile, globalización y expansión metropolitana: lo que existía sigue existiendo", EURE, 25, 1999, 29-56.

Dutta, Madhumita, "Place of life stories in labour geography: Why does it matter?" Geoforum, 77, 2016, $1-4$.

Fuentes, Luis \& Sierralta, Carlos, “Santiago de Chile, ¿ejemplo de una reestructuración capitalista global?”, EURE, 30, 2004, 7-28.

Goodman, Michael, Goodman, Daniel \& Redclift, Michael (eds), Consuming Space: Placing Consumption in Perspective, Ashgate, 2010.

Harvey, David, The Condition of Postmodernity: An Enquiry into the Origins of Cultural Change, Wiley-Blackwell, 1991.

Hauge, Mads \& Fold, Niels, "Resilience and reworking practices: Becoming the first-generation of industrial workers in Can Tho, Vietnam", Geoforum, 77, 2016, 124-133.

Jackson, Peter, "Local consumption cultures in a globalizing world", Sheffield, Transactions of the Institute of British Geographers, 29, 2004, 165-178.

Jones, Andrew \& Murphy, James, "Theorizing practice in economic geography: foundations, challenges, and possibilities", Progress in Human Geography, 35, 2010, 366-392.

Katz, Cindy, Growing up global: economic restructuring and children's everyday lives, Minneapolis, University of Minnesota Press, 2004.

Lastra, Facundo, "La superexplotación de la fuerza de trabajo y la especificidad del capitalismo latinoamericano: un aporte al debate, Izquierdas", 38, 2018, 257-278.

Mansvelt, Juliana, Geographies of Consumption, London, SAGE Publications, 2005.

Massey, Doreen, A global sense of place, Marxism Today, 1991.

Massey, Doreen "Power Geometry and Progressive Sense of Place", en John Bird, Barry Curtis, Tim Putnam y Lisa Tickner (eds.), Mapping the futures: Local culture, Global change, Nueva York, Routledge, 1993, 59-69.

Massey, Doreen Spatial Divisions of Labour: Social structures and the geography of production, London, Palgrave Macmillan, 1995.

Miller, Daniel,Jackson, Peter, Thrift, Nigel, Holbrook, Beverley \& Rowlands, Michael (eds.), Shopping, Place and Identity, London and New York, Routledge, 1998. 
Palma, Gabriel, “Chile 1914-1935: De economía exportadora a sustitutiva de importaciones”, en Oscar Muñoz (ed.), Perspectivas históricas de la economía chilena: del siglo XIX a las crisis del 30, Santiago, Colección Estudios CIEPLAN, 1984, 61-88.

Patton, Michael, Qualitative Research \& Evaluation Metods, Los Angeles, London, New Delhi, Singapore, Washington DC, Sage Publications, 2015.

Pumain, Denise \& Saint-Julien, Thérèse, Análisis espacial: Las interacciones, Santiago, Pontificia Universidad Católica de Chile y Universidad de Concepción, 2014.

Sandoval, Carlos, Especialización y concentración espacial de barrios comerciales: El caso de la comuna de Santiago, Santiago, Universidad de Chile, 2012.

Sepúlveda, Ulises "Recuperando la espacialidad de los sujetos: metodologias cualitativas para el análisis espacial, un modelo de topos, paisajes y tecnologias”. Investigaciones Geográficas, No 96 (2018).

Valdés, Ximena, Godoy, Carmen \& Mendoza, Angie, “Acción colectiva y resistencia: asalariadas agrícolas en Chile frente a la precarización laboral”, Izquierdas, 35, 2017, 167-198.

Wrigley, Neil, Retail Geographies, Southampton, Elsevier, 2009.

\section{Documentos públicos y privados}

Kassai, László, Cuero, calz̧ado y afines en Chile: ¿Una industria en desaparición o en búsqueda de un nuevo destino?, Santiago, CEPAL, 2000.

SERCOTEC, Evaluación de resultados intermedios de programa de fortalecimiento de barrios comerciales 2015-2017, 2017.

\section{Fuentes electrónicas o digitalizadas}

Oficios Varios, "El barrio Victoria", 22 de Agosto de 2014, disponible en: https://oficiosvarios.cl/elbarrio-victoria/

\section{Referencias orales (entrevistas)}

LVAC, Entrevista a locataria de local de venta de artículos confeccionados.

LVMC2, Entrevista a locatario de local de venta de materiales para la confección.

TCA, Entrevista a locatario de taller de confección de artículos.

TLVAC1, Entrevista a locataria de un taller y local de venta de artículos confeccionados.

TLVAC2, Entrevista a locatario de un taller y local de venta de artículos confeccionados. 